\title{
Development of Thermal Conductivity Prediction System for Composites*
}

\author{
Yibin Xu, Junichi Kinugawa and Koichi Yagi \\ Materials Information Technology Station, National Institute for Materials Science, Tokyo 153-0061, Japan
}

\begin{abstract}
For composites, it is possible to control their effective thermal conductivity through selecting the component materials and designing the structure. For this purpose, a computer system that can help a designer to find the optimum solution of materials and structure is necessary. In this work, we have developed a high-speed and light-weight thermal conductivity evaluation engine, basing on analytical solutions of effective thermal conductivity of composites. A package of calculation modules corresponding to the analytical solutions for different structure models is developed. As input data, the constitution of a composite is described by a file in XML data format. The engine analyzes the file, recognizes the structure model, and automatically selects a proper calculation module for the composite. The evaluation engine is connected to a materials database, which is used to stores and provides information of component materials. The calculation result of a designed composite can also be stored in the database for sharing and reusing. This system is expected to be used as an effective decision support tool for composites design.
\end{abstract}

(Received November 21, 2002; Accepted January 9, 2003)

Keywords: thermal conductivity, composite, prediction, design, analytical solution, materials database

\section{Introduction}

In most physical and chemical processes, heat transfer and temperature change occur. Thus, thermal conductivity, a fundamental property that dominates a material's behavior in heat and temperature transferring processes, must be taken into account, when the material's applicability is evaluated, especially for materials used as electronic devices, space materials, energy materials, etc.

For composites, it has been found that the effective thermal conductivity depends on the thermal conductivity, volume fraction and geometric distribution of each component. Therefore, it is possible to control the effective thermal conductivity of a composite through selecting the components and designing the structure. To help a designer to find the optimum solution, a computer system that can (1) predict the thermal conductivity of a designed composite; (2) show the tendency of thermal conductivity changing with the change of constitutional parameters; (3) store abundant information of candidate materials, is necessary.

In this work, we have developed a thermal conductivity prediction system, which is featured by a high-speed and light-weight calculation engine, and with capability to store and provide large amount of materials information.

\section{Thermal Conductivity Evaluation Methods}

The thermal conductivity of a composite can be calculated by solving the heat conduction equation $k \nabla^{2} T=0$ and $q=-k \nabla T$, where $k$ is the thermal conductivity, $q$ is the static heat flux and $T$ is the temperature. There are two ways to resolve this problem, numerical method and analytical method.

Almost all of the software products for resolving heat transfer problem are based on numerical method such as finite difference method (FDM) and finite element method (FEM). A numerical method focuses on analyzing the heat transfer and temperature distribution inside a specific

*This Paper was Presented at the Autumn Meeting of the Japan Institute of Metals, held in Suita, on November 3, 2002. composite. If any change of the constitution, such as volume fraction or geometry of a component, happens, the solution becomes invalid.

However, it is inconvenient to use a numerical method to study how the thermal conductivity changes with the change of constitution, because the designing and analyzing for each composite cost long time. And if we want to deal with a composite in which the dispersion phase are randomly distributed, the computing complexity of numerical analysis may become very high, which even makes this method impracticable.

Starting from Maxwell ${ }^{1)}$ and Rayleigh, ${ }^{2)}$ many theoretical studies $^{3--10)}$ have been done to analytically resolve the problem of conduction in heterogeneous materials. The law of mixtures, effective-medium theory ${ }^{3--6)}$ and equivalent inclusion method ${ }^{7,8)}$ have been widely adopted as effective methods of thermal conductivity evaluation for composites. ${ }^{11)}$

An analytical solution is usually deduced basing on one structure model, which usually includes some simplification, for example supposing the shape and size of dispersions are the same, and the distribution is uniform. An analytical solution can be applied to any composite as long as the structure model is valid. For example, a model of spherical dispersions will keep valid regardless the radius and density of the dispersion.

The basic structure models of composites are shown in Fig. 1. Generally, they can be divided into two categories: laminate composite and dispersion composite. For dispersion composites, according to the orientation of dispersion, they can be divided into three types: one dimensional (1D) orientation, two dimensional (2D) orientation and three dimensional (3D) orientation. The geometry of a dispersion can be approximately represented by an ellipsoid with semiaxes of $a, b$ and $c$, as shown in Fig. 2(a). When $a=b=c$, it represents $a$ sphere; and when $c$ tends to infinite, it represents an elliptical cylinder. The orientation of a dispersion is specified by two angles $\alpha$ and $\varphi$ as shown in Fig. 2(b). According to the maximum and minimum values of $\alpha$ and $\varphi$, we may judge which type the dispersion composite is. 

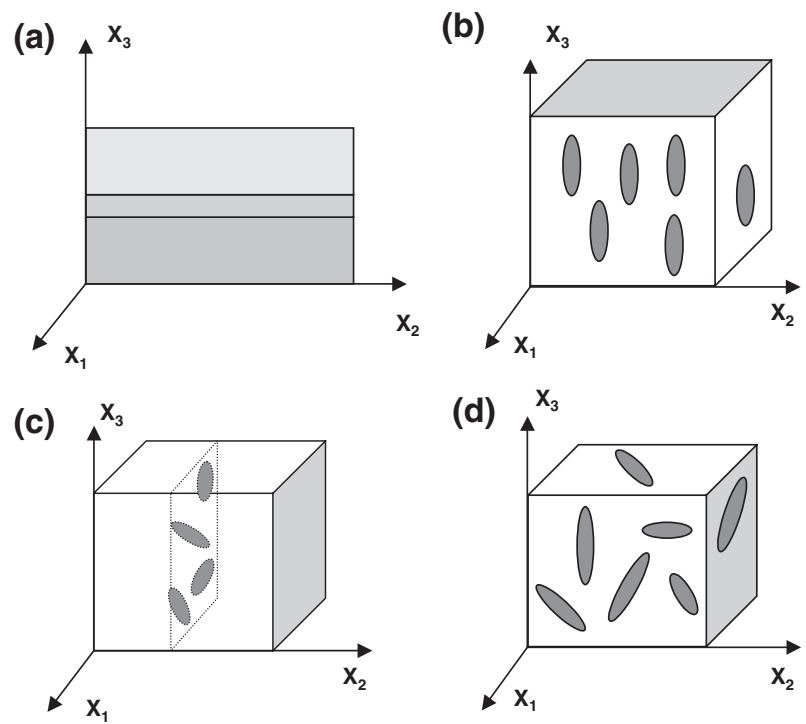

Fig. 1 Structure models of composites (a) laminate composite (b) dispersion composite with 1D orientation (c) dispersion composite with 2D orientation (d) dispersion composite with 3D orientation.

(a)

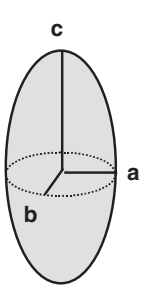

(b)

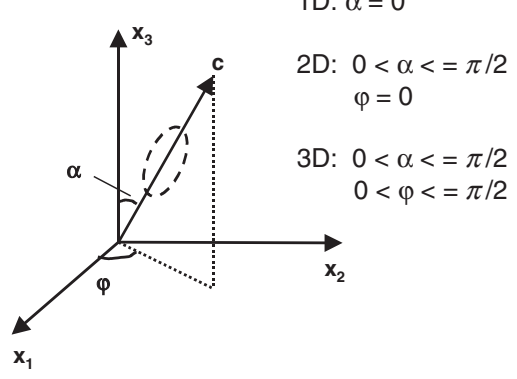

Fig. 2 Geometry (a) and direction (b) of a dispersion.

Table 1 Constitutional parameters that affect effective thermal conductivity of a composite.

\begin{tabular}{|c|c|}
\hline $\begin{array}{l}\text { Structure } \\
\text { model }\end{array}$ & Constitutional parameters \\
\hline Laminate & $\begin{array}{l}\text { Thermal conductivity of each layer; } \\
\text { Volume fraction of each layer }\end{array}$ \\
\hline Dispersion & $\begin{array}{l}\text { Thermal conductivity of matrix; } \\
\text { Thermal conductivity of dispersion; } \\
\text { Volume fraction of dispersion; } \\
\text { Geometry }(a, b, c) \text { of dispersion; } \\
\text { Orientation (maximum and minimum value of } \alpha \text { and } \varphi \text {, } \\
\text { respectively) of dispersion; } \\
\text { Thickness of interface between matrix and dispersion; } \\
\text { Thermal conductivity or thermal conductance } \\
\text { of the interface. }\end{array}$ \\
\hline
\end{tabular}

Analytical solutions express the effective thermal conductivity of composites as a function of the constitutional parameters, so it is an important guidance for optimization of constitution. Table 1 shows the constitutional parameters that affect the thermal conductivity in the case of laminate and dispersion composites, respectively.
Table 2 Analytical solutions of thermal conductivity and the corresponding structure models.

\begin{tabular}{|c|c|c|c|c|}
\hline \multicolumn{4}{|c|}{ Composites structure model } & \multirow{2}{*}{$\begin{array}{l}\text { Analytical } \\
\text { solution } \\
\text { reference }\end{array}$} \\
\hline Category & $\begin{array}{c}\text { Geometry of } \\
\text { dispersion }\end{array}$ & $\begin{array}{c}\text { Orientation of } \\
\text { dispersion }\end{array}$ & Interface & \\
\hline Laminate & - & - & No interface & $\begin{array}{l}\text { Law of } \\
\text { mixture }\end{array}$ \\
\hline Laminate & - & - & $\begin{array}{l}\text { Thin interface } \\
\text { (thickness } \\
\text { neglected) }\end{array}$ & $\begin{array}{l}\text { Hasselman } \\
\text { et al. }{ }^{9)}\end{array}$ \\
\hline Laminate & - & - & $\begin{array}{l}\text { Thick interface } \\
\text { (thickness } \\
\text { considered) }\end{array}$ & $\begin{array}{l}\text { Law of } \\
\text { mixture }\end{array}$ \\
\hline Dispersion & Ellipsoid & $\begin{array}{l}1 \mathrm{D}, 2 \mathrm{D} \text {, and } \\
3 \mathrm{D}\end{array}$ & No interface & Hatta et al. ${ }^{7)}$ \\
\hline Dispersion & Sphere & - & Thin interface & Hasselman \\
\hline Dispersion & Cylinder & $1 \mathrm{D}$ & $\begin{array}{l}\text { (thickness } \\
\text { neglected) }\end{array}$ & et al. ${ }^{9)}$ \\
\hline Dispersion & Cylinder & $1 \mathrm{D}$ & Thick interface & Markworth $^{6)}$ \\
\hline Dispersion & Ellipsoid & $1 \mathrm{D}$ & $\begin{array}{l}\text { (thickness } \\
\text { considered) }\end{array}$ & Hatta et al. ${ }^{8)}$ \\
\hline
\end{tabular}

The computing complexity of thermal conductivity calculation based on analytical solutions is much lower than that of numerical analysis, so it costs much less time. For commercial software of numerical analysis, it usually takes 20 to 30 minutes or even longer to solve the heat conduction equation, and then another process calculating the thermal conductivity is necessary. While with this system, the calculation of thermal conductivity can be finished within 1 second.

However, for different structure models, there are different analytical solutions. So we can not expect one calculation module being able to be used for all of the composites. In this work, a package of calculation modules has been developed, which includes the analytical solutions for all of the basic structure models in Fig. 1. The analytical solutions included in the package and their corresponding structure models are listed in Table 2.

\section{Description of Constitution and Properties of Com- posites}

To be able to select a proper calculation module for a composite, the evaluation engine must recognize its structure model. An input data file with indication of the structure model as well as the data of constitutional parameters is necessary.

Files in XML data format is used as input and output files. A XML file can be easily processed by a computer, and it is also a human-readable text file, which can be edited with any text editor.

Table 3 shows an example of the input and output files. The name of a data item is written as a tag, for example, $\langle$ volumeFraction $\rangle$. The value of the item lies between the start and end tags, for example, 〈volumeFraction〉 0.4 $\langle/$ volumeFracion $\rangle$. The thermal conductivity for each temperature point is expressed by three components $(k 11, k 22$ 
Table 3 An example of input and output files.

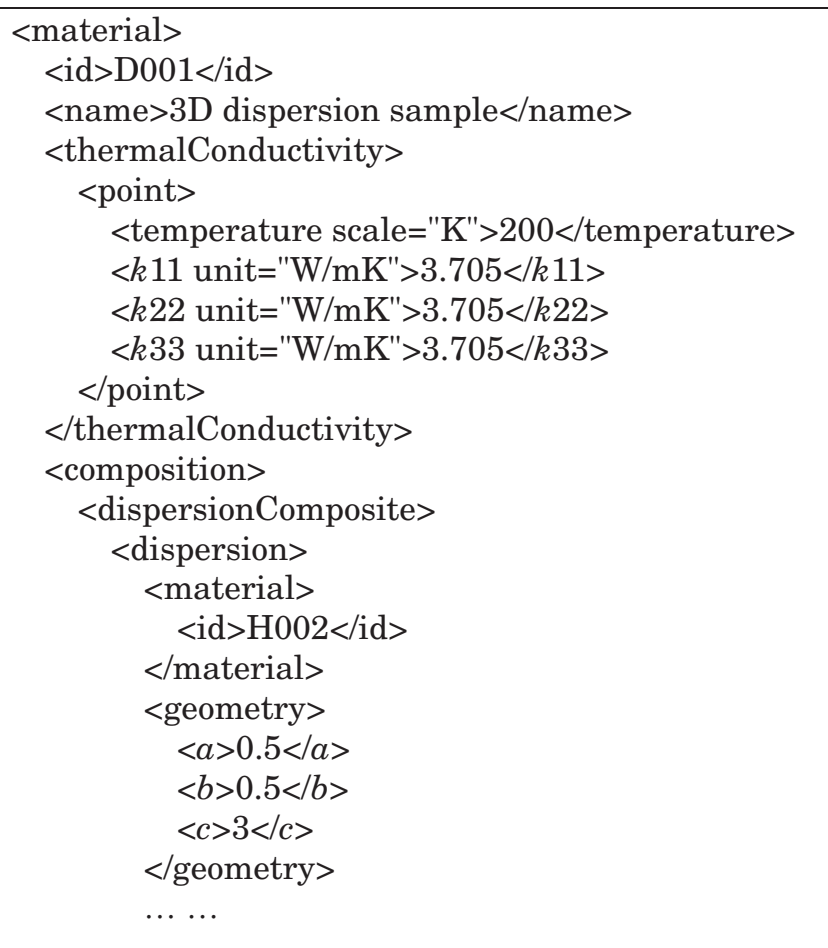

and $k 33$ ) along the axes of coordinates shown in Fig. 1. An input file only specifies the value of temperature, and leaves the values of thermal conductivity empty. After calculation, the calculated values are automatically inserted into the file.

A structure analyzer is developed to analyze the input XML file, recognizing the structure model, reading in the parameters, and selecting a proper calculation module according to the constitution described.

\section{Materials Database}

A materials database has been built inside this system, which can store abundant information of materials. It is helpful for users to find appropriate materials when design a composite, and provide the evaluation engine with the data required in calculation, such as thermal conductivity of components. The output files of the evaluation engine can also be saved in the database, so that the knowledge of composite design can be accumulated, and shared and reused by other users.

\section{System Architecture and Flow Chart}

The architecture of this system and flow chart of thermal conductivity calculation is shown in Fig. 3. The system is mainly composed by three parts, a structure analyzer, a package of calculation modules and a materials database.

An input XML file that describes the constitution of a composite is firstly analyzed by the structure analyzer, which recognizes the structure model, reads in the constitutional parameters, and selects an appropriate calculation module from the package. If the thermal conductivity of any component is not specified in the input file, the structure

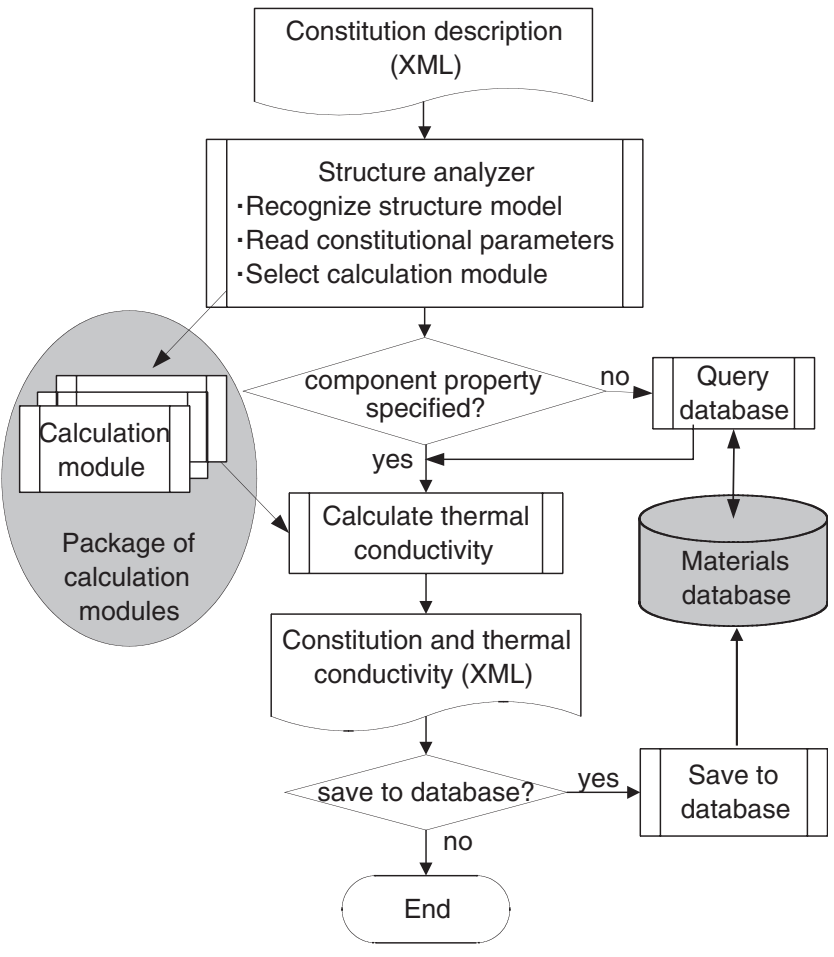

Fig. 3 Flow chart of thermal conductivity calculation.

analyzer queries the materials database to get it. After that, the thermal conductivity is calculated by the selected calculation module with the constitutional parameters. After calculation, the value of thermal conductivity is inserted into the XML file, and if the user want, it is saved to the database.

\section{Decision Support for Composites Design}

The system described above is expected to be used as an effective tool for designing a composite with expected thermal conductivity. Figure 4 shows an example of visualized calculation results for dispersion composites with 1D orientation. From it, we can clearly see how the effective thermal conductivity changes with change of the thermal conductivities of the components and the geometry of the dispersion.

\section{Conclusion}

A thermal conductivity prediction system for composites has been developed based on analytical solutions of heat conduction equations for heterogeneous materials. The system is featured by a high-speed and light-weight calculation engine, and a built-in materials database.

Our future work includes developing a web based user interface for materials selection and structure design, and making this system accessible from the internet, so that it can be used as a convenient and effective decision support tool for design of composites. 
(a)

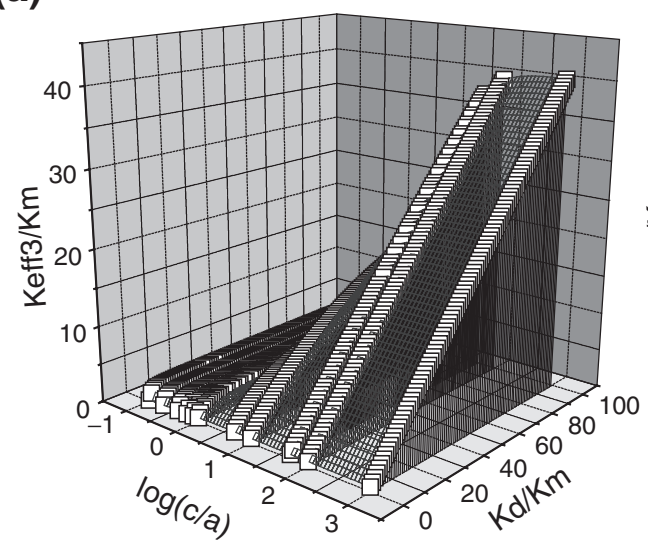

(b)

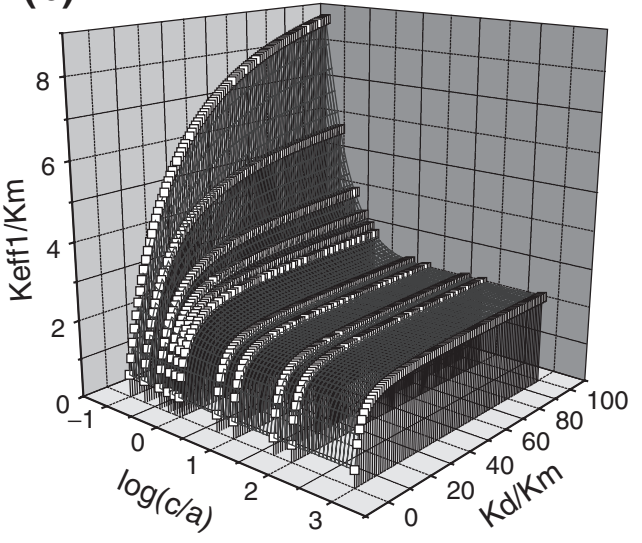

Fig. 4 Thermal conductivity of dispersion composites with 1D orientation. Here, c and a are semi-axes of the ellipsoid shown in Fig. 2, and with $a=b . K d$ and $K m$ are thermal conductivities of dispersion and matrix respectively. Keff 3 is the effective thermal conductivity along the $\mathrm{x}_{3}$ axis in Fig. 1(b), and Keff1 perpendicular to it.

\section{REFERENCES}

1) J. C. Maxwell: A treatise on electricity and magnetism, Vol. 1, 3rd edn (Oxford University Press, London, 1892) p. 435-449.

2) L. Rayleigh: Philos. Mag. 34 (1892) 481-502.

3) S. Kirkpatrick: Phys. Rev. Lett. 27 (1971) 1722-1725.

4) S. Kirkpatrick: Rev. Mod. Phys. 45 (1973) 574-588.

5) J. Bernasconi: Phys. Rev. B 9 (1974) 4575-4579.
6) A. J. Markworth: J. Mater. Sci. Lett. 12 (1993) 1487-1489.

7) H. Hatta and M. Taya: J. Appl. Phys. 58 (1985) 2478-2486.

8) H. Hatta and M. Taya: J. Appl. Phys. 59 (1986) 1851-1860.

9) D. P. H. Hasselman and Llord F. Johnson: J. Compod. Mater. 21 (1987) 508-515.

10) G. P. Marino: J. Nucl. Mater. 38 (1971) 178-190.

11) P. M. Hui, X. Zhang, A. J. Markworth and D. Stroud: J. Mater. Sci. 34 (1999) 5497-5503. 